\title{
BMJ Open Simulated presence therapy for dementia: a systematic review protocol
}

\author{
Iosief Abraha, ${ }^{1}$ Joseph M Rimland, ${ }^{1}$ Isabel Lozano-Montoya, ${ }^{2}$ \\ Giuseppina Dell'Aquila, ${ }^{1}$ Manuel Vélez-Díaz-Pallarés, ${ }^{2}$ Fabiana M Trotta, ${ }^{2}$ \\ Antonio Cherubini ${ }^{1}$
}

To cite: Abraha I, Rimland JM, LozanoMontoya I, et al. Simulated presence therapy for dementia: a systematic review protocol. BMJ Open 2016;6: e011007. doi:10.1136/ bmjopen-2015-011007

- Prepublication history and additional material is available. To view please visit the journal (http://dx.doi.org/ 10.1136/bmjopen-2015011007).

Received 29 December 2015 Accepted 15 January 2016

CrossMark

\begin{abstract}
${ }^{1}$ Department of Geriatrics, National Institute of Health and Science on Aging (INRCA), Ancona, Italy 2Department of Geriatrics, Hospital Universitario Ramón y Cajal, Madrid, Spain
\end{abstract}

Correspondence to Dr losief Abraha; iosief_a@yahoo.it

\section{ABSTRACT}

Introduction: The majority of patients with dementia develop behavioural and psychological symptoms of dementia (BPSD). Non-pharmacological interventions are an appealing alternative for the treatment of BPSD in patients with dementia. Simulated presence therapy (SPT) is a simple and inexpensive nonpharmacological intervention that can be used to treat BPSD. We propose a Cochrane protocol for the collection and assessment of evidence concerning the efficacy of SPT to treat relevant outcomes in people with dementia.

Methods and analysis: We will search the following electronic databases: the Cochrane Dementia and Cognitive Improvement Group's Specialised Register MEDLINE, EMBASE, CINAHL, PsycINFO, LILACS, CENTRAL and a number of trial registers as well as grey literature sources. We will include randomised and quasi-randomised controlled trials (including crossover studies) that evaluated SPT in people with dementia. Comparators such as usual care with no additional activity, or any activity that differs in content and approach from SPT, but is additional to usual care, will be considered. The primary outcomes of interest will comprise behavioural and psychological symptoms, as measured by relevant scales, and quality of life. Two review authors working independently and in tandem will be involved in title and abstract screening, full-text screening and data abstraction. Where possible, quantitative data will be pooled, and relative risk and mean difference with $95 \% \mathrm{Cl}$ will be employed for dichotomous and continuous data, respectively. Assessment of risk of bias will be performed using the Cochrane risk-of-bias tool and the Grades of Recommendation, Assessment, Development and Evaluation approach.

Ethics and dissemination: Ethics approval is not required. The final results of this systematic review will be presented to the Cochrane Library and will also be disseminated at relevant conference presentations.

Trial registration number: CRD42015029778.

\section{INTRODUCTION}

\section{Description of the condition}

Dementia is a common and serious neuropsychiatric syndrome, characterised by

\section{Strengths and limitations of this study}

- This review protocol aims to assess the evidence behind the use of simulated presence therapy for the treatment of relevant outcomes including behavioural and psychological symptoms in patients with dementia.

- The approach of the review will be a comprehensive search of the medical literature, followed by a transparent and systematic application of the Cochrane and Grades of Recommendation, Assessment, Development and Evaluation methods to evaluate the methodological quality of the evidence found.

- The results of this systematic review may help clinicians make decisions regarding simulated presence therapy.

- As in other non-pharmacological interventions for the treatment of behavioural disturbances in patients with dementia, we expect limitations of the evidence in terms of study design and execution, and small sample size.

progressive cognitive and functional decline. The prevalence of dementia is estimated to be $6 \%$ in populations aged 60 years or older. ${ }^{1}$ It increases dramatically with age, reaching $43 \%$ in people 85 years or older. ${ }^{2}$

Dementia imposes a considerable burden on families and is becoming a major socioeconomic challenge for the healthcare system. ${ }^{3}$ It is one of the strongest predictors of nursing home admission and is a major clinical issue for nursing home residents. Its prevalence varies from $26 \%$ to $48 \%$ in US nursing homes to over $60 \%$ in European nursing homes. ${ }^{4}$

The majority of patients develop behavioural disturbances, also known as behavioural and psychological symptoms of dementia (BPSD), over the course of the condition. BPSD comprise a heterogeneous group of non-cognitive symptoms, such as agitation, disruptive behaviour and aberrant motor behaviour including wandering, irritability, hallucinations, anxiety, depression, 
apathy, delusions, disinhibition and appetite, and sleep alterations. ${ }^{5}$ BPSD are a major component of the dementia syndrome as they are strongly correlated with the severity of functional impairment, occurring most frequently in people with dementia who are in hospitals and nursing homes. These symptoms are independently associated with poor outcomes such as distress among patients and caregivers, inappropriate prescribing of antipsychotic medication, long-term hospitalisation, mortality, excess morbidity, institutionalisation, early placement in a nursing home and increased healthcare costs. $^{6-9}$

Several studies have documented that almost all people with dementia experience at least one episode of behavioural disturbance at some point during their illness. ${ }^{10-13}$ The Cache County Study, in a cohort of 408 dementia participants, estimated the 5-year prevalence of neuropsychiatric symptoms and found that $97 \%$ of the participants experienced at least one symptom. Five-year prevalence was highest for depression $(77 \%)$, apathy $(71 \%)$ and anxiety (62\%). Rates for agitation/ aggression vary between $13 \%$ and $24 \% .{ }^{13}$ Behavioural symptoms frequently occur together (eg, wandering with sleep problems or irritability with persecutory delusions). ${ }^{14}$

\section{Description of the intervention}

Behavioural problems are often treated with drugs, which may lead to several adverse outcomes including sedation, falls, extrapyramidal disturbances, stroke and increased mortality. ${ }^{15-17}$ For this reason, non-pharmacological interventions are an appealing alternative. ${ }^{18}$

Several non-pharmacological interventions have been evaluated to treat BPSD in people with dementia. These include emotion-oriented therapies (such as reminiscence therapy ${ }^{19}$ simulated presence therapy ${ }^{20}$ and validation therapy ${ }^{21}$ ), sensory stimulation interventions (such as acupuncture, ${ }^{22}$ aromatherapy, ${ }^{23}$ light therapy, ${ }^{24}$ massage/touch therapy, ${ }^{25}$ music therapy, ${ }^{26}$ Snoezelen multisensory stimulation ${ }^{27}$ and transcutaneous electrical nerve stimulation ${ }^{28}$ ), and other interventions such as functional analysis-based interventions ${ }^{29}$ and exercise therapy. ${ }^{30}$

It has been reported that psychosocial interventions work best to reduce agitation and to improve other behavioural disturbances in people with dementia when they are personalised to reflect the individual's background and environmental circumstances. ${ }^{31}$

The focus of this review is simulated presence therapy (SPT), in which video or audiotape recordings of family members are played to the person with dementia. ${ }^{32}$ The content of the recordings may vary depending on the interests of the individual and may include conversations, stories or shared memories. The intention is that the recorded voice of a family member is reassuring, and that anxiety and distress are reduced by making the environment of the person with dementia as familiar as possible. $^{32}$

\section{How the intervention might work}

SPT is sometimes referred to as simulated family presence therapy, ${ }^{33}$ because it originated from the observation that nursing home residents who received more visits from family members were less agitated and had greater life satisfaction. ${ }^{32}$ Since family members may not be able to visit frequently, and family visits may be forgotten rapidly by residents with dementia, SPT aims to reduce the separation anxiety experienced by the patient. $^{3435}$

The technique was first described in a 1995 study by Woods and Ashley, ${ }^{32}$ in which 27 nursing home residents with dementia listened to a tape prepared by their caregivers. The authors reported a substantial reduction of behavioural problems such as verbal aggression and social isolation and, at the same time, an increase in positive behaviours such as proper verbalisation, smiling and singing. In the following years, a limited number of studies have been performed to evaluate the efficacy of SPT. These studies are characterised by considerable methodological heterogeneity, including different outcomes, as some studies examined the effect of SPT on quality of life, while other investigations attempted to reduce challenging behaviours.

The mechanisms by which SPT might work are still unknown.

Several explanatory models have been proposed for behavioural disturbances. According to the needdriven, dementia-compromised behaviour model, behavioural disturbances of the cognitively impaired person are an expression of an unmet need or goal. ${ }^{36}$ These disturbances might therefore be seen as an expression of the person seeking help or contact from family members.

Another conceptual framework, the progressively lowered stress threshold model, proposes that people with dementia become progressively unable to understand their environment (eg, incapable of recognising objects and their purposes and use). As a consequence, people with dementia experience stress that causes a variety of behavioural disturbances. ${ }^{37}$ In this context, voices and images of family members may represent well-known references that help to lower stress levels.

Generally, the literature is scarce in terms of the epidemiology of adverse events associated with nonpharmacological interventions. In the case of SPT, Woods and Ashley ${ }^{32}$ did not report any adverse events related to SPT. However, adverse events, including the possibility that the intervention might worsen agitation or mood, cannot be excluded. In some cases, for example, material contained in the tapes could be distressing for the patient. ${ }^{35}$

\section{Why it is important to conduct this review}

The majority of BPSD, in particular agitation, disruptive behaviour and aberrant motor behaviours, are currently treated with psychotropic drugs that are not very effective, ${ }^{38-40}$ have several important adverse effects including 
increased mortality, ${ }^{41}{ }^{42}$ and result in excessive drug costs and healthcare utilisation. ${ }^{43}{ }_{44}$ Non-pharmacological interventions should therefore be the first choice to treat BPSD.

SPT is quite a simple and inexpensive intervention. As such, we believe that it is important to verify whether SPT is effective in treating BPSD in people with dementia.

\section{Objectives}

To assess the effects of SPT on behavioural and psychological symptoms and quality of life in people with dementia.

\section{METHODS}

Criteria for considering studies for this review

Types of studies

We will include randomised and quasi-randomised controlled trials, including cross-over studies, of SPT in people with dementia.

\section{Types of participants}

People with any form of dementia diagnosed according to the International Classification of Diseases, Revision 10 (ICD-10), Diagnostic and Statistical Manual of Mental Disorders, Fourth Edition (DSM-IV), DSM-V or comparable criteria.

\section{Types of interventions}

SPT consisting of audio or video recordings that family members or caregivers have personalised. The content of the recording should include positive experience from the participant's past life and shared memories involving family or close friends.

Comparison:

- Usual care with no additional activity.

- Any activity that differs in content and approach from SPT, but is additional to usual care (eg, reminiscence groups, reality orientation groups, or social contact groups that do not use the techniques identified as SPT).

\section{Types of outcome measures}

\section{Primary outcomes}

1. Any behavioural and psychological symptoms as measured by scales such as: the Neuropsychiatric Inventory or Brief Psychiatric Rating Scale (multidomain scales), Cohen-Mansfield Agitation Inventory (scale specific to agitation) or Cornell Scale for Depression in Dementia (scale specific to depression)

2. Quality of life.

\section{Secondary outcomes}

1. Activities of daily living

2. Caregiver burden

3. Dropouts (as a measure of acceptability).
Search methods for identification of studies

Electronic searches

We will search ALOIS (http://www.medicine.ox.ac.uk/ alois), the Cochrane Dementia and Cognitive Improvement Group's Specialised Register.

ALOIS is maintained by the Trials Search Co-ordinator for the Cochrane Dementia and Cognitive Improvement Group and contains studies that fall within the areas of dementia prevention, dementia treatment and management, and cognitive enhancement in healthy elderly populations. The studies will be identified from inception to February 2016, through:

1. Monthly searches of a number of major healthcare databases: MEDLINE, EMBASE, CINAHL, PsycINFO and LILACS;

2. Monthly searches of a number of trial registers: ISRCTN, UMIN (Japan's trial register), the WHO International Clinical Trials Registry Platform (which covers ClinicalTrials.gov, ISRCTN, the Chinese Clinical Trials Register, the German Clinical Trials Register, the Iranian Registry of Clinical Trials and the Netherlands National Trials Register, plus others);

3. Quarterly search of the Cochrane Library's Central Register of Controlled Trials (CENTRAL);

4. Six-monthly searches of a number of grey literature sources: ISI Web of Knowledge Conference Proceedings, Index to Theses, Australasian Digital Theses.

To view a list of all sources searched for in ALOIS, see About ALOIS, on the ALOIS website (http://www. medicine.ox.ac.uk/alois).

Details of the search strategies run in healthcare bibliographic databases and used for the retrieval of reports of dementia, cognitive improvement and cognitive enhancement trials, can be viewed in the 'methods used in reviews' section within the editorial information about the Cochrane Dementia and Cognitive Improvement Group (CDCIG).

We will run additional searches in MEDLINE, EMBASE, PsycINFO, CINAHL, ClinicalTrials.gov and the WHO International Clinical Trials Registry Platform, to ensure that the searches for each suite of reviews is as comprehensive and up-to-date as possible. The search strategy we will use for the retrieval of reports of trials from MEDLINE (via the Ovid SP platform) was developed by the CDCIG and is available in online supplementary appendix 1 .

We will carry out translations for non-English papers, where possible.

\section{Searching other resources}

We will search the grey literature, such as conference proceedings and the reference lists of all the potentially relevant trials or reviews identified through the above search strategy. We will apply no language restriction. 


\section{Data collection and analysis}

Selection of studies

In order to facilitate the retrieval of articles of interest, we will download all references into a single database, using EndNote reference management software.

After removing duplicates, two pairs of review authors (JMR and IA; and IL-M and MV-D-P) will independently assess the titles and abstracts, when available, of all potentially relevant studies identified by the search strategy. We will obtain full-text copies, which will again be independently assessed by the two pairs of review authors. Any disagreements between review authors will be resolved by discussion or by a third review author (AC).

\section{Data extraction and management}

For eligible studies, two review authors (JMR and MV) will independently extract the data, using a standardised form. Any discrepancies will be resolved by discussion or by a third review author (AC).

We will attempt to extract the following information from each included study:

- General information about the study (year of publication, setting);

- Study protocol, where available;

- Study design, methods of recruitment, inclusion and exclusion criteria, details of the control and comparison groups and incentives for participation;

- Description and number of participants;

- Characteristics of the intervention;

- Results.

One review author will enter data into the Review Manager software (The Nordic Cochrane Centre TCC. Review Manager (RevMan) [Computer program]. Version 5.3. Copenhagen, 2014), and a second review author will check the data for accuracy.

\section{Assessment of risk of bias in included studies}

We will use The Cochrane Collaboration's recommended tool for assessing risk of bias. ${ }^{45}$

We will assess the following domains of each included study: sequence generation, allocation concealment, blinding of participants, personnel and outcome assessors, incomplete outcome data, selective reporting bias and other potential sources of bias. ${ }^{45}$ Other sources of bias will include the comparability of intervention and control group characteristics at baseline, validation of outcome assessment tools and reliability of outcome measures.

Two review authors will independently evaluate the risk of bias of the included studies, using the criteria outlined in the Cochrane Handbook for Systematic Reviews of Interventions. ${ }^{45}$ Any disagreements will be resolved by discussion or by a third review author.

We will describe the quality assessment in a 'Risk of bias' table.
Measures of treatment effect

Where we identify a sufficient number of trials with homogeneous populations, we will carry out meta-analyses of primary and secondary outcomes. For the analysis of dichotomous outcomes, we will use risk ratios with 95\% CIs; for the analysis of continuous outcomes, we will use the mean difference or standardised mean difference and $95 \%$ CI.

\section{Unit of analysis issues}

The unit of analysis will be the individual participant with dementia.

In case of studies with multiple correlated comparisons, we will combine study groups into a single pairwise comparison (ie, we will combine all appropriate experimental groups of the study into a single arm and all appropriate control groups into a single control arm).

In the event of cluster randomised trials, if the reported analysis does not correctly account for the cluster design, we will consider whether it is possible to estimate the effective sample size, using the intracluster correlation coefficient. If this is not possible, then we will conduct sensitivity analyses excluding the trials that did not originally adjust for clustering.

\section{Dealing with missing data}

In the event of missing data, we will contact the corresponding authors to try to obtain the relevant information.

We will not use any imputation methods. If intention-to-treat data are available, they would be preferred. If intention-to-treat data are not available, then we will conduct analyses using 'available cases' data.

\section{Assessment of heterogeneity}

We will assess heterogeneity of study characteristics and statistical heterogeneity. We will evaluate the former by examining the relative table of characteristics of the included population, the type of interventions and the type of outcome measures.

We will appraise statistical heterogeneity for each meta-analysis through a visual assessment of the forest plot, in addition to evaluating the $\chi^{2}$ test and $\mathrm{I}^{2}$ statistic. As recommended by Deeks et al, ${ }^{46}$ we will consider a $\chi^{2}$ test with a $p$ value of 0.10 to be significant, and we will interpret the $\mathrm{I}^{2}$ statistic as: $0-40 \%$ unimportant heterogeneity; 30-60\% moderate heterogeneity; $50-90 \%$ substantial heterogeneity and $75-100 \%$ considerable heterogeneity.

\section{Assessment of reporting biases}

Where there are more than 10 studies that can be combined into a meta-analysis, we will use a funnel plot to examine for small study effects, which may indicate publication bias. 


\section{Data synthesis}

We will pool data for analysis if the interventions, outcomes and participant populations in the individual studies are sufficiently similar (to be determined by consensus). We will not pool data for meta-analysis if we identify significant clinical, methodological or statistical heterogeneity.

We anticipate that interventions might differ from study to study, and consequently that different studies may be estimating different intervention effects. We will therefore initially perform analysis using a random-effects model. We will check the robustness of our conclusions to this choice by performing sensitivity analyses in which we use a fixed-effect model.

We will use Review Manager 5 for all analysis (The Nordic Cochrane Centre TCC, 2014).

\section{Subgroup analysis and investigation of heterogeneity}

Where sufficient data are available, we will perform subgroup analyses based on several participant characteristics such as sex, severity of dementia, severity of behavioural problems at baseline, the nature of the control intervention (treatment 'as usual' or another active treatment), stage of dementia, care setting (community vs long-term care facility) and intervention characteristics such as length of treatment, including number of sessions and mode of delivery (audio vs video recording).

\section{Sensitivity analysis}

If we include a sufficient number of studies in this review, we will perform sensitivity analyses to assess the consistency and robustness of the results of the meta-analyses. When sufficient data are available, we will perform sensitivity analysis based on:

- Including only studies with low-risk of selection bias (sequence generation and allocation concealment);

- Including only trials with low-risk of detection bias (blinding of outcome assessor);

- Including only trials with low-risk of attrition of bias;

- Including only trials that used an intention-to-treat analysis;

- Repeating analyses using a fixed-effect model for data synthesis.

\section{Summary of findings table}

We will use the Grades of Recommendation, Assessment, Development and Evaluation approach to assess the quality of the supporting evidence behind each estimate of treatment effect. We will use risk of bias, imprecision, inconsistency, indirectness and publication bias, to rate the overall evidence. ${ }^{47}$

We will present key findings of the review in a 'summary of findings' table. This will include a summary of the amount of data, the magnitude of the effect size and the overall quality of the evidence for the following outcomes: overall behavioural and psychological symptoms, agitation, depression, anxiety, quality of life and dropouts. ${ }^{48} 49$

\section{ETHICS AND DISSEMINATION}

Ethics approval is not required. The final results of this systematic review will be presented to the Cochrane Library and will also be disseminated at relevant conference presentations.

Acknowledgements The authors would like to thank Sue Marcus, the Managing Editor, and Jenny McCleery, Joint Co-ordinating Editor, Cochrane Dementia and Cognitive Improvement Group (CDCIG), for supporting the development of this protocol, and Anna Noel-Storr, Trials Search Co-ordinator, (CDCIG), for developing the search strategy.

Contributors IA and AC conceived the manuscript. IA, AC, JMR, MV-D-P, IL-M and GD drafted the manuscript and approved the final version of the manuscript.

Funding This manuscript was partly funded by the European Union Seventh Framework programme (FP7/2007-2013) under grant agreement $n^{\circ} 305930$ (SENATOR).

\section{Competing interests None declared.}

Provenance and peer review Not commissioned; peer reviewed for ethical and funding approval prior to submission.

Open Access This is an Open Access article distributed in accordance with the Creative Commons Attribution Non Commercial (CC BY-NC 4.0) license, which permits others to distribute, remix, adapt, build upon this work noncommercially, and license their derivative works on different terms, provided the original work is properly cited and the use is non-commercial. See: http:// creativecommons.org/licenses/by-nc/4.0/

\section{REFERENCES}

1. Prince M, Bryce R, Albanese E, et al. The global prevalence of dementia: a systematic review and metaanalysis. Alzheimers Dement 2013;9:63-75 e2.

2. Thies W, Bleiler L. 2012 Alzheimer's disease facts and figures. Alzheimers Dement 2012;8:131-68.

3. Wimo A, Jönsson L, Bond J, et al. The worldwide economic impact of dementia 2010. Alzheimers Dement 2013;9:1-11 e3.

4. Cherubini A, Ruggiero C, Dell'Aquila G, et al. Underrecognition and undertreatment of dementia in Italian nursing homes. J Am Med Dir Assoc 2012;13:759.e7-13.

5. Cerejeira J, Lagarto L, Mukaetova-Ladinska EB. Behavioral and psychological symptoms of dementia. Front Neurol 2012;3:73.

6. Borson S, Raskind MA. Clinical features and pharmacologic treatment of behavioral symptoms of Alzheimer's disease. Neurology 1997;48(Suppl 6):S17-24.

7. Clyburn LD, Stones MJ, Hadjistavropoulos T, et al. Predicting caregiver burden and depression in Alzheimer's disease. J Gerontol B Psychol Sci Soc Sci 2000;55:S2-13.

8. Kales HC, Gitlin LN, Lyketsos CG. Assessment and management of behavioral and psychological symptoms of dementia. BMJ 2015;350: h369.

9. Van Den Wijngaart MA, Vernooij-Dassen MJ, Felling AJ. The influence of stressors, appraisal and personal conditions on the burden of spousal caregivers of persons with dementia. Aging Ment Health 2007;11:626-36.

10. Kunik ME, Snow AL, Davila JA, et al. Causes of aggressive behavior in patients with dementia. $J$ Clin Psychiatry 2010;71:1145-52.

11. Lyketsos CG. Neuropsychiatric symptoms (behavioral and psychological symptoms of dementia) and the development of dementia treatments. Int Psychogeriatr 2007;19:409-20.

12. Spalletta G, Musicco M, Padovani A, et al. Neuropsychiatric symptoms and syndromes in a large cohort of newly diagnosed, untreated patients with Alzheimer disease. Am J Geriatr Psychiatry 2010;18:1026-35.

13. Steinberg $M$, Shao $H$, Zandi $P$, et al. Point and 5-year period prevalence of neuropsychiatric symptoms in dementia: the Cache County Study. Int J Geriatr Psychiatry 2008;23:170-7. 
14. Savva GM, Zaccai J, Matthews FE, et al. Prevalence, correlates and course of behavioural and psychological symptoms of dementia in the population. Br J Psychiatry 2009;194:212-19.

15. Lopez OL, Becker JT, Chang YF, et al. The long-term effects of conventional and atypical antipsychotics in patients with probable Alzheimer's disease. Am J Psychiatry 2013;170:1051-8.

16. Sink KM, Holden KF, Yaffe K. Pharmacological treatment of neuropsychiatric symptoms of dementia: a review of the evidence. JAMA 2005;293:596-608.

17. Stern $\mathrm{Y}$, Albert M, Brandt J, et al. Utility of extrapyramidal signs and psychosis as predictors of cognitive and functional decline, nursing home admission, and death in Alzheimer's disease: prospective analyses from the Predictors Study. Neurology 1994;44:2300-7.

18. Abraha I, Cruz-Jentoft A, Soiza RL, et al. Evidence of and recommendations for non-pharmacological interventions for common geriatric conditions: the SENATOR-ONTOP systematic review protocol. BMJ Open 2015;5:e007488.

19. Woods RT, Bruce E, Edwards RT, et al. Reminiscence groups for people with dementia and their family carers: pragmatic eight-centre randomised trial of joint reminiscence and maintenance versus usual treatment: a protocol. Trials 2009;10:64.

20. Zetteler J. Effectiveness of simulated presence therapy for individuals with dementia: a systematic review and meta-analysis. Aging Ment Health 2008;12:779-85.

21. Neal M, Barton Wright P. Validation therapy for dementia. Cochrane Database Syst Rev 2003;(3):CD001394.

22. Peng WN, Zhao H, Liu ZS, et al. Acupuncture for vascular dementia Cochrane Database Syst Rev 2007;(2):CD004987.

23. Forrester LT, Maayan N, Orrell M, et al. Aromatherapy for dementia. Cochrane Database Syst Rev 2014;2:CD003150.

24. Forbes D, Blake CM, Thiessen EJ, et al. Light therapy for improving cognition, activities of daily living, sleep, challenging behaviour, and psychiatric disturbances in dementia. Cochrane Database Syst Rev 2014;2:CD003946.

25. Viggo Hansen N, Jørgensen T, Ørtenblad L. Massage and touch for dementia. Cochrane Database Syst Rev 2006;(4):CD004989.

26. Vink AC, Birks JS, Bruinsma MS, et al. Music therapy for people with dementia. Cochrane Database Syst Rev 2004;(3):CD003477.

27. Chung JC, Lai CK, Chung PM, et al. Snoezelen for dementia. Cochrane Database Syst Rev 2002;(4):CD003152.

28. Cameron M, Lonergan E, Lee H. Transcutaneous electrical nerve stimulation (TENS) for dementia. Cochrane Database Syst Rev 2003;(3):CD004032.

29. Moniz Cook ED, Swift K, James I, et al. Functional analysis-based interventions for challenging behaviour in dementia. Cochrane Database Syst Rev 2012;2:CD006929.

30. Forbes D, Thiessen EJ, Blake CM, et al. Exercise programs for people with dementia. Cochrane Database Syst Rev 2013;12: CD006489.

31. O'Connor DW, Ames D, Gardner B, et al. Psychosocial treatments of psychological symptoms in dementia: a systematic review of reports meeting quality standards. Int Psychogeriatr 2009;21:241-51.

32. Woods P, Ashley J. Simulated presence therapy: using selected memories to manage problem behaviors in Alzheimer's disease patients. Geriatr Nurs 1995;16:9-14.

33. Garland K, Beer E, Eppingstall B, et al. A comparison of two treatments of agitated behavior in nursing home residents with dementia: simulated family presence and preferred music. Am J Geriatr Psychiatry 2007;15:514-21.

34. Miesen Bère ML. Alzheimer's disease, the phenomenon of parent fixation and bowlby's attachment theory. Int J Geriatr Psychiatry 1993;8:147-53.

35. Peak JS, Cheston RI. Using simulated presence therapy with people with dementia. Aging Ment Health 2002;6:77-81.

36. Algase DL, Beck C, Kolanowski A, et al. Need-driven dementia-compromised behavior: an alternative view of disruptive behavior. Am J Alzheimers Dis Other Demen 1996;11:10-19.

37. Smith M, Gerdner LA, Hall GR, et al. History, development, and future of the progressively lowered stress threshold: a conceptual model for dementia care. J Am Geriatr Soc 2004;52: 1755-60.

38. Howard R, McShane R, Lindesay J, et al. Donepezil and memantine for moderate-to-severe Alzheimer's disease. N Engl J Med 2012;366:893-903.

39. Katz I, de Deyn PP, Mintzer J, et al. The efficacy and safety of risperidone in the treatment of psychosis of Alzheimer's disease and mixed dementia: a meta-analysis of 4 placebo-controlled clinical trials. Int J Geriatr Psychiatry 2007;22:475-84.

40. Olin J, Schneider L. Galantamine for Alzheimer's disease. Cochrane Database Syst Rev 2002;(3):CD001747.

41. Budnitz DS, Lovegrove MC, Shehab N, et al. Emergency hospitalizations for adverse drug events in older Americans. $N$ Engl J Med 2011;365:2002-12.

42. Opie J, Rosewarne R, O'Connor DW. The efficacy of psychosocial approaches to behaviour disorders in dementia: a systematic literature review. Aust N Z J Psychiatry 1999;33:789-99.

43. Leendertse AJ, Van Den Bemt PM, Poolman JB, et al. Preventable hospital admissions related to medication (HARM): cost analysis of the HARM study. Value Health 2011;14:34-40.

44. Rottenkolber D, Schmiedl S, Rottenkolber M, et al. Adverse drug reactions in Germany: direct costs of internal medicine hospitalizations. Pharmacoepidemiol Drug Saf 2011;20:626-34.

45. Higgins JPT, Altman DG, Sterne JAC. Chapter 8: Assessing risk of bias in included studies. In: Higgins JPT, Green S, eds. Cochrane Handbook for Systematic Reviews of Interventions Version 5.1.0. The Cochrane Collaboration, 2011. http://wwwcochranehandbookorg2011

46. Deeks J, Higgins J, Altman D. Chapter 9: analysing data and undertaking meta-analysis. In: Higgins JPT, Green S, eds. Cochrane handbook for systematic reviews. Wiley-Blackwell, 2011:243-96.

47. Balshem $\mathrm{H}$, Helfand $\mathrm{M}$, Schunemann HJ, et al. GRADE guidelines: 3 . Rating the quality of evidence. J Clin Epidemiol 2011;64:401-6.

48. Schünemann HJ, Oxman AD, Higgins JPT, et al. Chapter 11: presenting results and 'Summary of findings' tables. In: Higgins JPT, Green S, eds. Cochrane handbook for systematic reviews of interventions. Version 5.1.0. The Cochrane Collaboration, 2011 http://wwwcochrane-handbookorg (accessed Mar 2015).

49. Schünemann HJ, Oxman AD, Vist GE, et al. Chapter 12: interpreting results and drawing conclusions. In: Higgins JPT, Green S, eds. Cochrane handbook of systematic reviews of interventions. Version 5.1.0. The Cochrane Collaboration, 2011. http://wwwcochranehandbookorg (accessed Mar 2015). 